\title{
APOYOS PARA AVANZAR HACIA UNA EDUCACIÓN MÁS INCLUSIVA EN LOS CENTROS ESCOLARES: ANÁLISIS DE GUÍAS PARA LA ACCIÓN
}

\section{Support in the advance towards more inclusive education in schools: an analysis of action guides}

\author{
Cecilia María Azorín Abellán \\ Universidad de Murcia \\ cmaria.azorin@um.es \\ Marta SAndoval Mena \\ Universidad Autónoma de Madrid
}

Recepción: 27 de septiembre de 2018

Aceptación definitiva: 1 de febrero de 2019

Resumen: El objetivo del estudio que se presenta fue realizar una revisión de las guías que ofrece la literatura en el campo de la investigación educativa para apoyar a los centros escolares en el desarrollo de prácticas más inclusivas. Así pues, el texto recoge el análisis de contenido llevado a cabo sobre un compendio de trece guías (publicadas mayoritariamente en lengua inglesa) que trata los siguientes aspectos: el propósito por el que fueron creadas, la perspectiva de inclusión de la que parten, el colectivo al que se dirigen, la etapa en la que tienen mayor funcionalidad, las estrategias para la acción que proponen, la estructura básica sobre la que se asientan, las dimensiones y los indicadores sobre los que estimulan la reflexión y las herramientas e instrumentos que plantean para la evaluación. Esta aproximación de naturaleza cualitativa ha permitido indagar en los pasos que estos documentos recomiendan a los centros educativos para emprender el denominado viaje hacia la inclusión. Las conclusiones apuntan a la necesidad de utilizar y dar a conocer el contenido de este tipo de recursos en contextos de habla hispana, generando espacios de reflexión y debate que contribuyan al avance de este activo pedagógico innegociable.

Palabras Clave: guías; educación inclusiva; mejora escolar; análisis documental. 
ABSTRACT: The aim of this study was to review the guides available in the educational research literature that support the development of more inclusive practices. The paper, therefore, reports on the analyses of a compendium of thirteen guides (mainly published in English) that address the following aspects: the purposes for which they were created, the perspective of inclusion they take as their starting point, the target readership, the stage at which they are most functional, the action strategies proposed, the basic structure on which they are founded, the dimensions and indicators used to stimulate reflection and the evaluation tools and instruments put forward. The approach is of a qualitative nature and enables us to enquire into the steps these papers recommend education centers should take in their journeys towards inclusion. The conclusions point to the need to make use of and to make known these types of resources in the Spanish speaking world by generating spaces for reflection and debate that will help to advance in this field.

KEY WORDS: guides; inclusive education; school improvement; documentary analysis.

\section{Transformar la realidad: el desafío de la inclusión}

P REPARAR A LA JUVENTUD PARA UN MUNDO INCLUSIVO Y SOSTENIBLE es uno de los objetivos prioritarios de la agenda educativa contemporánea (OECD, 2018). Sin embargo, cómo hacer que las sociedades y, por ende, las escuelas sean más inclusivas no es una cuestión de fácil respuesta. En términos de inclusión, si lo que se pretende es transformar la realidad, hay que dar pequeños pasos, orientar el trayecto y proporcionar palancas de cambio que permitan a esta revolución pendiente (revolución inclusiva) tener lugar con garantías de éxito. Así pues, coincidimos con otros colegas que posicionan a la educación inclusiva como una aspiración y perspectiva desde la que analizar los desafíos que comporta la equidad en la educación (Ainscow, 2015; Echeita, 2017; Messiou, 2017).

Nos encontramos hoy en día con el desafío de llegar a todos los procesos y sistemas de prácticas (Puig-Rovira et al., 2012), para que configuren y determinen una nueva gramática escolar que sostenga con firmeza el compromiso con los valores y los principios de la educación inclusiva (Booth y Ainscow, 2015).

Es preciso comprender que la inclusión no es una tendencia de moda más en la investigación educativa, sino una obligación que tienen los organismos, administradores y responsables de las políticas escolares, que han de escuchar las demandas de la ciudadanía y revertir los efectos perversos de un sistema educativo que no termina de responder con garantías a todo su alumnado. Cabe afirmar que no solo en España, sino también en otros países dentro y fuera de Europa, crece lo que hemos venido a denominar "despotismo inclusivo", término acuñado para determinar la perpetuación del “todo por la inclusión, pero sin la inclusión”, un planteamiento que refrenda el discurso de la teoría que cabalga, por un lado, y la realidad de la práctica que lo hace, por otro, muy diferente. Por su parte, Slee (2013) plantea el dilema de cómo hacer que la educación inclusiva suceda cuando la exclusión es una predisposición política. No obstante, es cierto que en los últimos años ha habido atisbos de avance e iniciativas 
llevadas a cabo con la finalidad de transformar las escuelas y las aulas en entornos de aprendizaje más inclusivos.

Somos conscientes, igual que Escudero (2012), que muchos aspectos están fuera del alcance de las instituciones, pero otros tantos son decisiones de las escuelas. De igual manera, Murillo y Hernández (2011) reflexionan que, si bien una institución puede ser el principal organismo de reproducción y legitimación de desigualdades sociales, también puede constituir el principal motor para el cambio social. En consecuencia, la inclusión, entendida esta como palanca de cambio social, es una de las principales fuerzas precursoras de la reforma educativa mundial, así como un objetivo central de la política internacional y el mayor reto que tienen por delante los centros escolares hoy en día. En este sentido, la Agenda 2030 para el Desarrollo Sostenible de las Naciones Unidas compromete a los diferentes países miembros a asegurar una educación inclusiva y de calidad, junto con la promoción de oportunidades de aprendizaje permanente para todos y todas (UNESCO, 2015a). En esta encrucijada de caminos, en la que transformar la realidad es el verdadero desafío de la inclusión, surge el interrogante acerca de cómo pueden las escuelas responder de manera efectiva a la diversidad y avanzar en una dirección más inclusiva (Azorín, 2016; Florian y Beaton, 2017; López-Vélez, 2018; Loreto, López y Assaél, 2015; Messiou y Ainscow, 2015; Miles y Ainscow, 2011; Simón, Echeita y Sandoval, 2018).

El concepto de inclusión ha sido ampliamente abordado, entre otros, por Ainscow et al. (2006), quienes lo entienden como un proceso que trata de identificar y suprimir barreras; que incorpora la presencia, participación y logro de todos los estudiantes sin excepción; e implica un énfasis particular en aquellos y aquellas que se encuentran en riesgo de exclusión, marginalización o bajo rendimiento. En el ámbito nacional e internacional existe acuerdo en torno a que la inclusión educativa es un derecho de todos los alumnos y alumnas, pero también hay consenso acerca de las trabas que obstaculizan a los sistemas y comunidades educativas para hacer efectivo este derecho. Inexorablemente, la construcción de sistemas educativos que garanticen la calidad educativa con equidad equivale a decir que la primera llegue a todos los niños, jóvenes y adultos a lo largo de la vida (Life Long Learning), sin estar condicionada por razones de salud, procedencia, lugar de residencia, etnia, capacidad económica, género, orientación afectivo-sexual o cualquier otra casuística.

Como venimos exponiendo, la educación inclusiva es un asunto relevante y ampliamente debatido en el panorama escolar dentro y fuera de nuestras fronteras. Sin embargo, es preciso matizar que la inclusión no surgirá de forma natural a partir del orden social existente (Göransson y Nilhom, 2014; Marchesi y Martín, 2014), sino como resultado de acciones conscientes, reflexivas y voluntarias.

En este sentido, es necesario arrojar luz a los equipos directivos y docentes de los centros educativos sobre el sentido y significado que comparten acerca de la inclusión, y las acciones que podrían tomar para mover las políticas y las prácticas en una dirección más inclusiva. Ya que nuestra experiencia práctica señala que la inclusión es entendida y asumida de forma distinta por parte de profesores, investigadores, familias y estudiantes.

(C) Ediciones Universidad de Salamanca / CC BY-NC-ND

Siglo Cero, vol. 50 (3), n. ${ }^{\circ}$ 271, 2019, julio-septiembre, pp. 7-27 


\section{Guías para emprender “el viaje” a la inclusión}

Han sido muchos los autores en el campo de la investigación educativa que han utilizado la metáfora del viaje para referirse a la trayectoria que deben recorrer las escuelas al iniciar procesos de cambio y transitar por otros espacios más inclusivos, lo que exige cuestionar tanto los valores y las políticas de las sociedades actuales (Messiou, 2012; Nguyen, 2015), como la capacidad de las escuelas para responder a la diversidad (Azorín y Ainscow, 2018; Echeita, 2006). En consonancia con lo expresado, diferentes evidencias que provienen de la teoría y práctica escolar, empiezan a aportar las lecciones aprendidas en el viaje hacia la inclusión de las escuelas españolas (Azorín, 2018b; Simón, Sandoval y Echeita, 2017). Si bien, conviene recordar que:

No es deseable un certificado que sugiera que la escuela ha alcanzado un destino final en cuanto a la inclusión. Las escuelas siempre están cambiando; los estudiantes y el personal llegan y se marchan; aparecen nuevas formas de exclusión; se movilizan nuevos recursos. La inclusión es un proceso sin final, "una historia interminable”. En el único sentido en el que sería deseable proclamar a una escuela como "inclusiva” es cuando se compromete firmemente con la sostenibilidad de un proceso de mejora escolar guiado por valores inclusivos (Booth y Ainscow, 2015: 31).

En relación con el análisis de las guías, teniendo como foco de indagación preferente el ámbito de la inclusión, existen investigaciones previas que han profundizado en aspectos tales como la autoevaluación y la mejora de la atención a la diversidad en los centros educativos (Guirao y Arnaiz, 2014); la evaluación y el apoyo para el seguimiento del proceso hacia la inclusión (Muntaner, 2016); así como la revisión de instrumentos sobre atención a la diversidad para una educación inclusiva de calidad (Azorín, Arnaiz y Maquilón, 2017). En este sentido, debe reconocerse el impacto y la influencia mundial que ha tenido el Index for Inclusion (Booth y Ainscow, 2011), herramienta formada por indicadores, orientaciones y preguntas de reflexión que tratan de apoyar a los equipos docentes para llevar a la práctica los principios y valores que deben sostener una educación para todas y todos. En relación con este instrumento, se ha afirmado lo siguiente:

Se trata de un instrumento útil para guiar la autorreflexión, la participación y el diálogo entre los diferentes miembros de la comunidad educativa. La finalidad última de esta guía es la de construir espacios educativos que promuevan la participación e incrementen su capacidad para responder a la diversidad del alumnado garantizando la equidad y calidad (Booth, Simón, Sandoval, Echeita y Muñoz, 2015: 5).

De igual modo, según se explora en la parte empírica de este trabajo, son numerosos los recursos que, inspirados por el Index, han aparecido en la literatura especializada como documentos de apoyo para la promoción de una escuela y una sociedad más inclusiva. En síntesis, el proceso hacia la educación inclusiva que está teniendo lugar en los centros de Educación Infantil, Primaria y Secundaria precisa de instrumentos, materiales de apoyo y guías que permitan orientar el camino, que proporcionen 
pautas para la autorreflexión y el análisis de la realidad y que, por consiguiente, favorezcan la puesta en marcha de propuestas de cambio y mejora inclusivas.

Este artículo aborda, per se, un tema de interés profesional para el avance de la inclusión en los centros escolares. A diferencia de lo ya investigado por otros colegas anteriormente, se expone a continuación una selección de guías (incluyendo dentro de este concepto materiales, documentos, recursos y herramientas) cuya finalidad es apoyar a las escuelas para ponerse en acción en lo que a cuestiones de inclusión se refiere. Consideramos que este compendio de recursos puede ayudar a los centros educativos a saber cómo dar los primeros pasos y en qué dimensiones fijar su atención para ponerse en marcha, asumiendo como condición sine qua non que la inclusión (y la atención a la diversidad) no es solo un desafío, sino también un derecho y un activo pedagógico innegociable.

\section{Objetivos}

El propósito general de la investigación que ha dado lugar a este trabajo fue realizar una revisión de las guías que ofrece la literatura para orientar y acompañar a los centros educativos en el desarrollo de prácticas más inclusivas. Por lo que se presentarán una serie de guías de ámbito nacional e internacional que facilitan procesos de evaluación en los centros, dirigidos a la mejora educativa. Para ello, nos centraremos en describir aquellas que gravitan sobre el ámbito específico de la atención a la diversidad, omitiendo otra serie de instrumentos que tratan aspectos muy específicos orientados a la inclusión de colectivos tradicionalmente vulnerables.

De forma específica, se planteó acometer el estudio de las guías seleccionadas atendiendo a los siguientes parámetros: objetivo por el que se crea,perspectiva de inclusión de la que parte, colectivo al que se dirige, etapa en la que tiene mayor funcionalidad, estrategias que propone para la acción, estructura básica sobre la que se asienta, dimensiones e indicadores que formula y herramientas e instrumentos que presenta para la puesta en marcha de una filosofía escolar inclusiva en la instituciones educativas.

\section{Método}

Se ha desarrollado una investigación de corte cualitativo en la que se describe el contenido de una serie de guías que han sido diseñadas para apoyar a las escuelas en su camino hacia la inclusión. Así pues, puede afirmarse que todas ellas tratan de ayudar a las instituciones educativas en el desarrollo de prácticas más inclusivas. El método aplicado fue el análisis documental de contenido (Barbosa, Barbosa y Rodríguez, 2013; Fernández, 2002; López, 2002; Peña y Pirela, 2007; Rojas, 2011). En este estudio se han elegido las guías más actuales (últimos 10 años) que introducen perspectivas significativas en este campo de conocimiento y que recogen el testigo de la realidad en la que vivimos. Precisamente, uno de los aspectos que en ocasiones generan contradicción a este respecto es lo efímeras que pueden llegar a resultar en el tiempo este tipo de herramientas, puesto que las políticas, los valores y los conocimientos 
evolucionan y cambian en gran medida de una década a otra. Esto hace que, salvo instrumentos de gran renombre por su impacto y valía en el contexto escolar, como es el caso del Index for Inclusion (una obra inmaterial que inspira a todas las demás), la mayoría de estos recursos precisan actualizaciones o cambios profundos para responder a las necesidades que plantea el contexto socioeducativo actual.

Por consiguiente, en la Tabla 1 se recogen por orden cronológico las 13 guías/ herramientas que fueron objeto de análisis en aras de conocer los pasos que estos documentos recomiendan para emprender procesos relacionados con la inclusión. Esto se ha hecho partiendo de guías que han sido ideadas para la mejora de la educación inclusiva en las escuelas y que pueden, por tanto, actuar como elementos catalizadores de la inclusión.

\begin{tabular}{|c|c|c|}
\hline & Guías & Autoría \\
\hline 1 & $\begin{array}{l}\text { Inclusion and Diversity in Education. Guidelines } \\
\text { for Inclusion and Diversity in Schools }\end{array}$ & British Council (2010) \\
\hline 2 & $\begin{array}{c}\text { Creating an Inclusive school. Indicators of } \\
\text { Success. A Reflection Tool for Administrators, } \\
\text { Educators and Other School Staff }\end{array}$ & $\begin{array}{l}\text { New Brunkwick Association for } \\
\text { Community Living (2011) }\end{array}$ \\
\hline 3 & $\begin{array}{l}\text { Inclusive Practice in Secondary Schools. Ideas for } \\
\text { School Leaders }\end{array}$ & $\begin{array}{c}\text { New Zealand Ministry of } \\
\text { Education (2014) }\end{array}$ \\
\hline 4 & $\begin{array}{l}\text { Coaching to Support Inclusion: A Principal’s } \\
\text { Guide }\end{array}$ & $\begin{array}{l}\text { The Alberta Teachers' Association } \\
\text { (2015) }\end{array}$ \\
\hline 5 & $\begin{array}{l}\text { Embracing Diversity: Toolkit for Creating } \\
\text { Inclusive, Learning-friendly Environments }\end{array}$ & UNESCO (2015b) \\
\hline 6 & How good is our school? & Education Scotland (2015) \\
\hline 7 & $\begin{array}{c}\text { Tool to Upgrade Teacher Education Practices for } \\
\text { Inclusive Education }\end{array}$ & Council of Europe (2015) \\
\hline 8 & $\begin{array}{c}\text { Equality: Making it Happen. A guide for schools } \\
\text { to make sure everyone is safe, included and } \\
\text { learning }\end{array}$ & $\begin{array}{l}\text { Centre for Studies on Inclusive } \\
\text { Education (2015) }\end{array}$ \\
\hline 9 & $\begin{array}{l}\text { The IB guide to inclusive education: a resource for } \\
\text { whole school development }\end{array}$ & $\begin{array}{l}\text { International Baccalaureate } \\
\text { Organization (2015) }\end{array}$ \\
\hline 10 & $\begin{array}{c}\text { Diversity, Equality and Inclusion Charter and } \\
\text { Guidelines for Early Childhood Care and } \\
\text { Education }\end{array}$ & $\begin{array}{l}\text { Department of children and youth } \\
\text { affairs (2016) }\end{array}$ \\
\hline 11 & $\begin{array}{l}\text { Reaching Out All Learners. A Resource Pack for } \\
\text { Supporting Inclusive Education }\end{array}$ & IBE and UNESCO (2016) \\
\hline 12 & $\begin{array}{c}\text { Guía para asegurar la inclusión y la equidad en la } \\
\text { educación }\end{array}$ & $\begin{array}{l}\text { Disponible en español e inglés } \\
\text { (UNESCO, 2017) }\end{array}$ \\
\hline 13 & Themis Inclusion Tool & $\begin{array}{l}\text { Disponible en inglés (Azorín y } \\
\text { Ainscow, 2018) y español (Azorín, } \\
\text { 2018a) }\end{array}$ \\
\hline
\end{tabular}


Las fuentes documentales propiamente dichas fueron las guías, herramientas y recursos seleccionados para la descripción de su contenido. En la fase de preselección se tuvieron en cuenta los siguientes criterios: 1) antigüedad: textos publicados en la última década; 2) temática: contenido vinculado con la atención a la diversidad y la educación inclusiva; 3) panorama nacional e internacional: visión de la inclusión dentro y fuera de nuestras fronteras; 4) guias preferentemente orientadas al apoyo de la inclusión en las escuelas (no diseñadas específicamente para la autoevaluación de prácticas, sino para el acompañamiento de los centros educativos en el camino hacia la inclusión); 5) carácter no tanto de naturaleza empírica sino reflexiva, abogando por la idea de inclusión no solo como una cuestión de números o estadística, sino como un elemento próximo a la realidad cualitativa, relacional y contextual que presentan los centros educativos, una perspectiva que entendemos que se encuentra inherentemente ligada al movimiento inclusivo, y 6) textos destinados a ayudar a los centros a situarse en su casilla de partida para el desarrollo de estrategias y planes de acción que favorezcan procesos educativos y sociales más inclusivos.

El análisis de los diferentes documentos se realizó a través de un cuadro de registro que permitió el vaciado y la recolección de información de manera estandarizada (Tabla 2).

\begin{tabular}{|c|l|}
\hline \multicolumn{1}{|c|}{ TABLA 2. Cuadro de registro y descripción de los aspectos recogidos en las guías } \\
objeto de análisis
\end{tabular}




\begin{tabular}{|c|l|}
\hline $\begin{array}{c}\text { TABLA 2. Cuadro de registro y descripción de los aspectos recogidos en las guías } \\
\text { objeto de análisis (cont.) }\end{array}$ \\
\hline $\begin{array}{c}\text { Modelo de cuadro para el análisis } \\
\text { dimensiones/ } \\
\text { indicadores }\end{array}$ & $\begin{array}{l}\text { Dimensiones e indicadores en los que ponen el foco de atención las } \\
\text { diferentes guías revisadas. }\end{array}$ \\
\hline $\begin{array}{c}\text { Estructura básica de } \\
\text { la guía }\end{array}$ & $\begin{array}{l}\text { Partes de las que se compone el documento para una mejor compren- } \\
\text { sión de su contenido. }\end{array}$ \\
\hline $\begin{array}{c}\text { Instrumentos para la la } \\
\text { revisión de prácticas }\end{array}$ & $\begin{array}{l}\text { En este apartado se recoge información sobre los instrumentos/ma- } \\
\text { teriales/recursos para la revisión de prácticas que las guías brindan } \\
\text { o ponen a disposición del lector. Estos instrumentos tratan de ayu- } \\
\text { dar/guiar a los centros educativos a la hora de ponerse en acción. Se } \\
\text { distingue entre rúbricas, cuestionarios, escalas, entrevistas, grupos de } \\
\text { discusión y actividades de reflexión, entre otros. }\end{array}$ \\
\hline
\end{tabular}

Una vez formulados los objetivos de la investigación y definidos los criterios de revisión, se procedió a la realización de una búsqueda bibliográfica teniendo en consideración los pasos propuestos por Guirao-Goris, Olmedo y Ferrer (2008): 1) consulta de bases de datos y fuentes documentales, 2) establecimiento de la estrategia de búsqueda, 3) especificación de los criterios de selección de documentos y 4) organización de la información. Para la búsqueda bibliográfica, se utilizaron las siguientes bases de datos: Web of Science, Dialnet, DicE, Scopus, así como la herramienta Google Scholar, que resultó útil para la localización de los textos, tanto en lengua inglesa como en español. Los descriptores o palabras clave utilizados fueron los siguientes: "guías de educación inclusiva", "herramientas para la inclusión" y su traducción al inglés: "inclusive education guides" y "tools for inclusion". Asimismo, se acotó la selección de fuentes documentales publicadas en la última década, acordando mantener como filtro el período que comprende desde el año 2010 hasta la actualidad. Paralelamente, se puso especial atención en las guías publicadas por organismos comprometidos con la inclusión, como la UNESCO.

\section{Resultados}

A pesar de la heterogenidad de países donde se han publicado y promovido los documentos que vamos a presentar a continuación, la aproximación terminológica de todas las guías revisadas es muy similar, especialmente por ser la mayoría producidas en países angloparlantes. En todas ellas emerge la constante de que la educación inclusiva es una combinación de filosofía y prácticas pedagógicas que permiten que cada estudiante se sienta respetado y seguro para que pueda aprender y desarrollar todo su potencial, basado en un sistema de valores y creencias compartido por la comunidad escolar.

Todos estos materiales constituyen oportunidades para "ponerse en acción", aunque es posible que algunas escuelas no sientan que están preparadas o quieran llevar a

(C) Ediciones Universidad de Salamanca / CC BY-NC-ND

Siglo Cero, vol. 50 (3), n. ${ }^{\circ} 271,2019$, julio-septiembre, pp. 7-27 
cabo una revisión completa de sus prácticas, pero sí pueden utilizar estas herramientas para estimular la reflexión, la discusión y el aprendizaje sobre la inclusión.

A continuación, se expondrán los aspectos más importantes de cada documento, haciendo explícitas las dimensiones, factores o indicadores que contemplan cada una de estas guías cuando se refieren a la educación inclusiva y en la forma de utilización de cada una de ellas.

1. Inclusion and Diversity in Education. Guidelines for Inclusion and Diversity in Schools [Inclusión y diversidad en la educación. Pautas para la inclusión y la diversidad en las escuelas] (British Council, 2010)

Esta guía se dirige a toda la comunidad escolar, especialmente a los equipos directivos y docentes tanto de escuelas de Primaria como de Secundaria. Propone un modelo de escuela culturalmente inclusiva, basado en investigaciones internacionales que incorpora buenas prácticas desarrolladas en el marco del proyecto INDIE ${ }^{1}$. Contiene tres dimensiones: marco legal que garantice igualdad de oportunidades; politicas nacionales sobre atención a la diversidad; y contexto escolar, que a su vez comprende los siguientes factores: liderazgo culturalmente inclusivo, altas expectativas para todos y todas, celebración de la diversidad, promoción de la innovación y el cambio, desarrollo de un currículo inclusivo, participación de la familia y empoderamiento de la voz de los estudiantes.

El proceso de mejora escolar se basa en la autoevaluación y la automejora y debe involucrar a todos los grupos que tienen una participación o interés en la escuela. Este se desarrolla en tres fases: auditoría, procedimientos de autoevaluación para detectar fortalezas y áreas de mejora, lo que implica acordar objetivos (estableciendo criterios mensurables), así como planificar las acciones; seguimiento del proceso, las escuelas tendrán reuniones regulares para valorar el desarrollo de los planes de acción, compartir el aprendizaje y revisar cualquier aspecto que no esté en el buen camino o los recursos no apropiados; y evaluación del progreso, mediante las evidencias encontradas fruto de las observaciones, entrevistas y utilización de cuestionarios.

2. Creating an Inclusive school. Indicators of Success. A Reflection Tool for Administrators, Educators and Other School Staff [Creando una escuela inclusiva. Indicadores de éxito. Una herramienta de reflexión para administradores, educadores y otro personal escolar] (New Brunkwick Association for Community Living, 2011)

La guía persigue motivar a las comunidades escolares de Educación Infantil, Primaria y Secundaria para promover prácticas inclusivas. Se trata de una herramienta de

1 El proyecto INDIE está patrocinado y administrado por el British Council e involucra a once países de Europa occidental divididos en tres grupos. De cada país se identifican 2-5 escuelas, con 10-20 líderes jóvenes y un coordinador que cuenta con el apoyo de la autoridad educativa relevante (nacional, regional o local). 
reflexión y acción que permite a los educadores y administradores conocer las claves de éxito en la creación y mantenimiento de centros inclusivos. Entre los indicadores "de éxito" que propone destacan los siguientes: respeto de las diversas experiencias, perspectivas y conocimientos de los estudiantes, así como del sentido de pertenencia; experiencias de aprendizaje inclusivas (programa de educación flexible, fortalezas y capacidades, entornos de aprendizaje comunes y participación plena, estudiantes fuera del entorno de aprendizaje común, plan de estudios, instrucción, evaluación en curso); apoyos disponibles para que el alumnado pueda participar plenamente; comportamiento (con incidencia en temas de acoso escolar); gestión y liderazgo proactivo de la escuela (uso eficaz de los recursos del entorno); innovación y ambiente creativo en el proceso educativo; y enfoque colaborativo para la búsqueda de soluciones y proyección hacia una comunidad escolar comprometida.

Los pasos que formula son los siguientes: clarifica el objetivo; articula los principios $y$ valores; determina quién participará e identifica a los facilitadores; diseña el proceso; desarrolla un plan de trabajo; implementa la propuesta, y evalúa los resultados. Entre los instrumentos que proporciona se incluyen cuestionarios para el profesorado, las familias y los adminsitradores educativos que favorecen la reflexión y análisis acerca del perfil que presenta la escuela.

3. Inclusive Practice in Secondary Schools. Ideas for School Leaders [Práctica inclusiva en escuelas de Secundaria. Ideas para líderes escolares] (New Zealand Ministry of Education, 2014)

El objetivo de esta guía es ofrecer un marco común de ideas para discutir en las escuelas de secundaria con inspectores de educación, directores y orientadores. La guía se organiza en cuatro dimensiones que a su vez constituyen rúbricas de evaluación: construir una cultura escolar inclusiva, esto comporta un debate acerca de las distintas creencias individuales y colectivas sobre la inclusión; desarrollar procesos y sistemas, se deben recoger datos y aspectos vinculados con el proceso de desarrollo de cada estudiante y los períodos de transición. Esto suele hacerse a través de una plataforma web, labor que puede desarrollar el departamento o grupo de agentes educativos; asesorar a aprendices diversos, puesto que hay que asegurar tanto la presencia como la participación y el aprendizaje (en este sentido se tiene que dar más importancia al cómo se aprende que al qué se aprende, es decir, a los procesos en lugar de los resultados); mejorar la colaboración y el compañerismo, para sostener todo este sistema y mejorarlo es muy importante la colaboración con las familias, así como con otras organizaciones y miembros de la comunidad. La voz de las familias y su papel en la toma de decisiones es fundamental.

El ciclo de investigación que propone para la mejora escolar a través de la metáfora del "viaje" incluye tres pasos: reunir al equipo bumano que va a formar parte de las discusiones; recabar información, y hacer un plan para cambiar y transformar las prácticas inclusivas en la escuela. 
APOYOS PARA AVANZAR HACIA UNA EDUCACIÓN MÁS INCLUSIVA EN LOS CENTROS ESCOLARES:

ANÁLISIS DE GUÍAS PARA LA ACCIÓN

CECILIA MARÍA AZORÍN ABELLÁN Y MARTA SANDOVAL MENA

4. Coaching to Support Inclusion: A Principal's Guide [Coaching para apoyar la inclusión: una guía para el director] (The Alberta Teachers' Association, 2015)

Esta guía persigue explorar el uso del coaching como estrategia de desarrollo profesional para apoyar la puesta en marcha de prácticas inclusivas tanto en centros de Primaria como de Secundaria. Los directores se convierten en formadores que trabajan con los docentes para satisfacer las necesidades de todos los estudiantes dentro de un entorno escolar inclusivo. Este recurso se crea para guiar y apoyar la inclusión por parte de los equipos directivos que han de trabajar en colaboración con el personal de la escuela para que esto suceda. En este sentido, resulta sumamente importante el establecimiento de una cultura colaborativa con roles definidos. La guía expone un conjunto de estrategias para mejorar la colaboración: crear una cultura de expectativas; aumentar el intercambio de información, y fomentar la transferencia de ideas e inquietudes.

A su vez, esta guía detalla las dimensiones que están asociadas al ejercicio de un liderazgo inclusivo en el que se propone: fomentar relaciones efectivas, representar un liderazgo visionario, liderar una comunidad de aprendizaje, proporcionar liderazgo instruccional, desarrollar y facilitar el liderazgo, administrar recursos escolares y comprender y responder al contexto social más amplio. Por último, establece tres fases claramente diferenciadas: movilización, consiste en actividades de preparación, creación de conciencia, construcción de compromiso y planificación del programa; implementación del plan, e institucionalización, en esta fase el coaching se convierte en parte de la cultura escolar del centro.

5. Embracing Diversity: Toolkit for Creating Inclusive, Learning-friendly Environments [Abrazando la diversidad: Conjunto de herramientas para crear entornos inclusivos y amigables para el aprendizaje] (UNESCO, 2015b)

Esta guía nació tras celebrarse la Conferencia Mundial de Educación en Dakar, que aspiraba a asegurar en el año 2015 el acceso de colectivos vulnerables de estudiantes a una educación de calidad. Fue escrita en el año 2000 y ha sido revisada en varias ocasiones hasta que en el año 2015 estuvo disponible su versión on line. Pretende sensibilizar a los futuros y actuales docentes y administradores educativos de cualquier etapa educativa formal o no formal sobre la importancia de la educación inclusiva y les proporciona herramientas prácticas para analizar su situación.

Cabe destacar que uno de los conceptos más relevantes que se acuña en esta guía es el término "inclusive, learning-friendly environment" (ambiente amistoso de aprendizaje inclusivo). Este concepto enfatiza la importancia de que los estudiantes y los maestros aprendan juntos como una comunidad de aprendizaje, situando a los estudiantes en el centro de su aprendizaje. Los apartados que cubre esta guía son los siguientes: construcción de un entorno inclusivo y amigable para el aprendizaje (incluyendo los beneficios para el profesorado, el alumnado, las familias y las comunidades) e identificación de aquellas áreas que pueden necesitar más mejoras, proporcionando ideas sobre cómo 
planificar cambios y cómo evaluar el progreso; trabajo con familias y comunidades describe cómo ayudar a las familias y otros miembros de la comunidad y organizaciones a participar en el desarrollo y mantenimiento de un entorno de aprendizaje inclusivo; que todos los niños y niñas vayan a la escuela y aprendan promueve la reflexión sobre las barreras que impiden que esto suceda, así como ejemplos de estudiantes que no pueden aprender ni participar; crear un ambiente de aprendizaje inclusivo, enfatizando la autoestima de los estudiantes y la necesaria unión del aprendizaje en casa y en la escuela; gestión de aulas de aprendizaje inclusivo, donde se tratan aspectos relacionados con la planificación de la enseñanza y el aprendizaje, el aprovechamiento de los recursos disponibles, la gestión del trabajo en grupo, el aprendizaje cooperativo y formas de evaluación coherentes con esta forma de aprender; y creación de un ambiente saludable $y$ protector, en lo que se refiere a políticas protectoras de la infancia, prevención de violencia, programas de nutrición escolar y servicios e instalaciones de salud.

6. How good is our school? [¿ Cómo de buena es nuestra escuela? (Education Scotland, 2015)

La guía se dirige a toda la comunidad educativa de centros de Educación Secundaria. Plantea un cambio en el que pueden intervenir todos los agentes implicados en el centro, así como grupos de personas relacionadas en el ámbito de la educación, como personal universitario o expertos.

El marco de mejora de la escuela consiste en un conjunto de 15 indicadores de calidad diseñados para ayudar a responder tres preguntas relacionadas con aspectos importantes del trabajo y la vida de la escuela. Los indicadores de calidad se dividen en tres categorías: liderazgo y gestión, que responde a la pregunta ¿cómo de bueno es nuestro liderazgo y enfoque de mejora?; disposición de aprendizaje, ¿qué tan buena es la calidad de la atención y educación que ofrecemos?, y éxitos y logros, ¿cuán buenos somos para garantizar los mejores resultados posibles para todos nuestros estudiantes?

Este material expone un modelo de autoevaluación que permite revisar las políticas y las prácticas. Asimismo, profundiza en procesos básicos para la gestión del cambio desde una triple panorámica mirando: hacia adentro (autoevaluación); hacia fuera, aprendiendo de lo que sucede en otros lugares; y hacia delante, explorando qué puede deparar el futuro para los estudiantes de hoy. En este modelo se enfatiza la necesidad de triangular la información mediante procedimientos de observación directa, recogida de datos cuantitativos e incorporación de todas las voces de la comunidad educativa.

7. Tool to Upgrade Teacher Education Practices for Inclusive Education [Herramienta para mejorar las prácticas de formación docente para la educación inclusiva] (Council of Europe, 2015)

Se trata de un recurso dirigido a estudiantes de grados, profesores en ejercicio y formadores de docentes que tienen el propósito de mejorar las prácticas escolares 
especialmente en centros de Secundaria. Esta herramienta consta de dos partes. La primera parte describe el procedimiento de actualización como una secuencia de actividades. Orienta a los docentes a través de un ciclo de resolución de problemas con seis etapas: identificación de problemas; evaluación de necesidades, metas y objetivos; estrategias educativas; implementación; evaluación, y comentarios. La segunda parte describe el marco para las prácticas inclusivas que está basado en los postulados de la Agencia Europea sobre el perfil de los docentes inclusivos. Este marco identifica cuatro prácticas relevantes para el desarrollo de habilidades asociadas con la educación inclusiva orientadas a ser un profesional competente en educación inclusiva; valorar la diversidad de los estudiantes; apoyar a todos los estudiantes, y utilizar estrategias de trabajo colaborativo.

El profesorado puede usar esta herramienta para comprender mejor su posición en su propio desarrollo profesional y las habilidades que pueden necesitar para progresar hacia un profesional inclusivo a través de un ciclo de resolución de problemas. En este sentido, señalan que el ciclo no es lineal ni secuencial sino que engloba procesos dinámicos e interactivos. Además, la guía en cuestión ofrece rúbricas con diferentes niveles de habilidad sobre competencias docentes tanto para estudiantes de magisterio, como profesores en ejercicio y directores/inspectores de centros educativos.

8. Equality: Making it Happen. A guide for help schools to make sure everyone is safe, included and learning [Igualdad: hacer que suceda. Una guia para ayudar a las escuelas a asegurarse de que todos estén seguros, incluidos y aprendiendo] (Centre for Studies on Inclusive Education, 2015)

Se trata de una guía práctica de fácil manejo para ayudar a las escuelas de Primaria y Secundaria a promover la igualdad y garantizar que todos los estudiantes se sientan seguros, visibles $y$, en definitiva, aprendan a convivir en igualdad.

Los indicadores en los que la guía centra su atención son los siguientes: discapacidad (incluidas las dificultades de aprendizaje), género e identidad de género, orientación sexual, culturaletnia, religión o creencias, embarazo y maternidad y origen socioeconómico. Junto a estos aspectos, se profundiza también en ambiente de aprendizaje, liderazgo, comportamiento, bienestar, logro, aprendizaje sobre igualdad, diversidad y derechos humanos, legislación británica en materia de igualdad, igualdad LGTB en educación, igualdad de discapacidad en educación, igualdad étnica en educación y aumento del logro de todos los alumnos y alumnas. Los materiales incluyen cuestionarios que se dirigen a alumnado, familias y personal docente para el seguimiento de la igualdad en el centro escolar.

Mediante ejemplos prácticos de experiencias en centros educativos del Reino Unido, la guía hace un breve recorrido por temáticas que están relacionadas con la inclusión. Plantea casuísticas concretas y expone cómo se han resuelto determinados problemas o de qué manera se han eliminado las barreras a través de procesos de diálogo y del uso, entre otros instrumentos, del propio Index for Inclusion. La guía propone procesos reflexivos y materiales de apoyo y consulta a partir de testimonios 
que provienen de la práctica. Contiene una serie de apartados que abordan distintos contenidos sobre igualdad de forma sencilla e incluye ejemplos de buenas prácticas y fuentes de información adicionales.

9. The IB guide to inclusive education: a resource for whole school development [La guia de educación inclusiva del IB: un recurso para el desarrollo de toda la escuela] (International Baccalaureate Organization, 2015)

Realizada por la agrupación de escuelas de Educación Primaria y Secundaria internacionales de la asociación Internacional Baccalaureate Organization (conocida como IB). La guía se dirige a directores, consultores y personal de apoyo. En primer lugar, reconoce el importante carácter contextual que tiene la inclusión y admite que las escuelas pueden encontrarse en diferentes puntos de desarrollo. Su objetivo principal es ayudar a las escuelas a estructurar y desarrollar prácticas de educación inclusiva.

La guía focaliza su atención en la participación de la comunidad, la política inclusiva y el desarrollo de una política escolar para la inclusión. Esta última se diferencia de la anterior en el uso de un ciclo de investigación, acción y reflexión que sostiene el desarrollo y la revisión de la política de inclusión en la propia escuela. Según este recurso, la creación de escuelas eficaces e inclusivas depende de la creación de entendimientos comunes en toda la comunidad escolar. Las escuelas deben considerar cómo incluir a todos los miembros de la comunidad a través de las siguientes acciones: creación de entornos de aprendizaje óptimos; el uso de la tecnología; el desarrollo de procesos de colaboración; enfoques inclusivos para el aprendizaje; evaluación, y enseñanza de la variabilidad (diferenciación y diseño universal del aprendizaje).

La propuesta para llevar a cabo los procesos de mejora consta de una serie de declaraciones que reflejan los ideales de inclusión con preguntas de autoevaluación. Son preguntas abiertas en las que no hay respuestas correctas o incorrectas. Tampoco hay recomendaciones de tiempo y las escuelas deben entender que el desarrollo de la educación inclusiva es un proceso a largo plazo. Asimismo, se incide en que si una escuela decide usar el proceso inclusivo de autorrevisión, debe ser integrado a las iniciativas y estrategias de desarrollo de toda la escuela. El ciclo de revisión ha de estar respaldado por el ciclo de investigación del $\mathrm{IB}$, donde la investigación, la acción y la reflexión involucran a toda la comunidad escolar desde un enfoque constructivista conducente a procesos abiertos y democráticos.

10. Diversity, equality and inclusion charter and guidelines for early childhood care and education [Carta de diversidad, igualdad e inclusión y pautas para el cuidado y la educación de la primera infancia] (Department of Children and Youth Affairs, 2016)

Esta guía se dirige a los profesionales de la Educación Infantil y resulta reveladora por su posicionamiento en el que defiende que la inclusión y la calidad van de la mano.

(C) Ediciones Universidad de Salamanca / CC BY-NC-ND

Siglo Cero, vol. 50 (3), n. ${ }^{\circ} 271,2019$, julio-septiembre, pp. 7-27 
Se señala que es fundamental apoyar a los niños y las niñas a construir identidades positivas, desarrollar un sentido de pertenencia y darse cuenta de su potencial. Además, es la única guía de todas las que aquí se presentan en la que se discute el concepto de los "fondos de conocimiento" y de "múltiples identidades", que trata de respetar la identidad única de los niños y las niñas en el nacimiento y su papel en la construcción y reconstrucción del significado personal dentro de sus contextos culturales.

Se reflexiona sobre distintas diversidades como la diversidad cultural, la adquisición de la segunda lengua, el género, las creencias religiosas y las comunidades de familias temporeras. En cada uno de estos temas se exponen las visiones que deberían tener las escuelas para respetar las diversidades y se aportan una serie de preguntas para ayudar a reflexionar a los educadores sobre las mencionadas cuestiones. Consta de dos partes, en la primera se hace mención al Estatuto Nacional Irlandés de la inclusión (de la primera infancia), que tiene como objetivo promover los valores de la diversidad, igualdad e inclusión. La segunda parte contiene directrices para la atención y el cuidado de la primera infancia, refiriéndose a los fondos de conocimiento y de identidad, enfoque educativo contra el sesgo, modificación del entorno físico, apoyo a las familias y análisis de la implementación de las políticas y liderazgo en las escuelas.

La forma propuesta para desarrollar la mejora en los centros se divide en siete pasos: decidir quién desarrollará; evaluar la política actual; generar un borrador de la política consultando a todas las partes interesadas; compartir el borrador preliminar con el personal, los voluntarios y las familias; ratificar la politica; implementar la política, y revisar la política.

\section{Reaching Out All Learners. A Resource Pack for Supporting Inclusive Education [Alcanzando a todos los estudiantes. Un paquete de recursos para apoyar la educa- ción inclusiva] (IBE y UNESCO, 2016)}

Es una guía dirigida a formadores, administradores educativos, docentes, equipos directivos de escuela, tanto de Primaria como de Secundaria. Hasta el momento, todas las guías promovidas por la UNESCO que se han analizado comparten supuestos teóricos similares. En este caso, se considera que la inclusión es un proceso relacionado con la identificación y la eliminación de barreras. La inclusión tiene tres dimensiones: presencia, participación y logro de todos los estudiantes. La inclusión implica un particular énfasis en aquellos grupos de aprendices que pueden estar en riesgo de marginación, exclusión y bajo logro. La forma en que invita a reflexionar es a través de textos de distinta naturaleza: resúmenes, proyectos de investigación educativos, estudios de casos de escuelas, preguntas de discusión y actividades.

La herramienta se organiza en tres secciones/subguías interconectadas, como se denomina en la publicación: una orientada a la política; otra a los centros educativos, y otra a las aulas. En cada una de ellas se ofrecen experiencias de escuela de todo el mundo, temas de discusión y actividades.

En síntesis, ofrece un marco de cuatro dimensiones (conceptos, política, estructura y práctica) con indicadores basados en la investigación educativa internacional. 
APOYOS PARA AVANZAR HACIA UNA EDUCACIÓN MÁS INCLUSIVA EN LOS CENTROS ESCOLARES:

ANÁLISIS DE GUÍAS PARA LA ACCIÓN

CECILIA MARÍA AZORÍN ABELLÁN Y MARTA SANDOVAL MENA

Finalmente, cuenta con una escala compuesta por las citadas dimensiones que permite evaluar las escuelas de forma genérica.

\section{Guía para asegurar la inclusión y la equidad en la educación (UNESCO, 2017)}

Esta guía es útil para docentes y equipos directivos de las etapas Primaria y Secundaria. En su contenido se advierte que la inclusión no solo se consigue facilitando el acceso a la educación, sino también proporcionando espacios de aprendizaje de calidad, y pedagogías que permitan a los estudiantes prosperar, comprender sus realidades y diferencias individuales como una oportunidad para democratizar y enriquecer el aprendizaje.

Además, presenta ejemplos concretos y ofrece un marco de revisión con indicadores para conocer el nivel de progreso de cada uno de ellos en cuatro dimensiones: concepto; políticas declaradas; estructuras y sistemas, y prácticas. Cada dimensión tiene, a su vez, cuatro características definitorias que pueden servir de autoevaluación de los sistemas, obtenida de la anterior publicación de la IBE y UNESCO (2016).

Esta herramienta hace hincapié en la necesidad de los docentes de renovarse y seguir aprendiendo constantemente para poder desarrollar una educación inclusiva y, para ello, establece cuatro valores centrales que desarrollan esa competencia docente: valorar la diversidad del alumnado, viendo las diferencias de los estudiantes como un recurso y un activo para la educación; apoyar a todos los estudiantes, teniendo altas expectativas en términos de logro para todos los estudiantes; trabajar con otros, considerando la colaboración y el trabajo en equipo como enfoques esenciales, y desarrollo profesional personal continuo, entendiendo la enseñanza como una actividad de aprendizaje en la que el personal docente debe aceptar la responsabilidad de su propia formación y actualización permanente.

\section{Themis Inclusion Tool [Herramienta de Inclusión Themis] (Azorín y Ainscow, 2018)}

Esta guía se dirige al personal directivo y docente que trabaja en centros de Educación Primaria y Secundaria. Su estructura está formada por tres dimensiones: contextos, recursos y procesos (inspirada en el Modelo CIPP de Stufflebeam). El uso de esta herramienta promueve la reflexión sobre los contextos donde están inmersos los centros educativos; los recursos con los que cuentan para atender a la diversidad del alumnado; y los procesos (inclusivos o no) que se llevan a cabo a este respecto en las escuelas.

Los indicadores en los que profundiza son los siguientes: situación socioeconómica; diversidad cultural; politica educativa; liderazgo; valores; prevención de discriminaciones; relación profesorado-alumnado; colaboración del profesorado; enlaces de la familia y el centro escolar; participación de la comunidad; redes de colaboración; recursos formativos, bumanos, materiales, tecnológicos, físicos y comunitarios, celebración de la diversidad; planificación de la enseñanza; proceso educativo; variedad 
metodológica; heterogeneidad y flexibilidad de los grupos-clase; organización de espacios y tiempos; apoyos; evaluación, y tránsito entre etapas educativas.

En este caso, los pasos que sugiere para revisar la práctica escolar están claramente influenciados por las fases desarrolladas en el propio Index for Inclusion: iniciar un proceso de reflexión en grupos de discusión que permita pensar sobre el sentido y significado de inclusión que comparten las partes interesadas y resolver las posibles contradicciones; cumplimentar el cuestionario individualmente para cotejar esta información con los datos recogidos en la fase anterior de manera grupal; analizar los resultados e identificar las fortalezas y debilidades, y seleccionar las lineas de cambio indicando aquellas prioridades sobre las que se pretende avanzar para la puesta en marcha de proyectos de mejora tendentes al desarrollo de prácticas más inclusivas. Un ejemplo de su aplicabilidad en centros educativos puede encontrarse en Azorín (2018a).

\section{Consideraciones finales}

A modo de cierre, cabe destacar que la mayoría de las guías revisadas enfatizan la importancia de aprender de los miembros de la comunidad educativa en un proceso de aprendizaje mutuo y de naturaleza reflexiva en cada contexto educativo. En esta dirección, el trabajo de Azorín y Ainscow (2018) apuesta por desarrollar instrumentos de revisión teniendo en cuenta los contextos particulares y las ideas de todas las personas involucradas en el viaje a la inclusión. Por tanto, los centros y sus comunidades educativas son los protagonistas de los cambios que no solo se plantean en las prácticas concretas de aula, sino también en la cultura (valores) y en la política de los mismos. No obstante, hay que ser conscientes del peso de la representación que tiene cada agente en estos documentos (en muchos de ellos, las familias y los estudiantes no se mencionan). Del mismo modo, no podemos ser ajenos a los conflictos y tensiones de diversa índole (personales, sociales o profesionales) que pueden aparecer como consecuencia de plantear cambios en los centros educativos. A veces esto supone, entre otras cosas, cuidar a los profesionales, lograr que se sientan valorados y que participen en las decisiones que les afectan.

El análisis desarrollado en este trabajo aporta evidencia sobre el estado actual de la temática investigada, así como información que puede ser de utilidad en procesos formativos y de trabajo en los contextos escolares. Tras la revisión realizada puede afirmarse que las distintas guías estudiadas favorecen procesos de apoyo, asesoramiento y acompañamiento en la propia acción, lo que hemos asumido como el camino o viaje hacia la inclusión. Indudablemente, este tipo de recursos permiten orientar el rumbo de los centros educativos que tratan de incorporar cambios dentro y fuera de las aulas para avanzar hacia el desarrollo de prácticas más inclusivas. Si bien, es fácil entender que cuando un centro escolar (sobre todo su profesorado, sus familias y su alumnado), no comparte -cada uno a su nivel- ciertos valores inclusivos, estamos frente a una realidad en la que difícilmente podrán florecer prácticas educativas que acojan, respeten y valoren la diversidad. Durante la revisión de estas guías se han apuntado algunos de 
esos valores inclusivos, como la igual dignidad de todos y con ella la ética del cuidado por aquellos más vulnerables; el reconocimiento de la diversidad y la responsabilidad de actuar para hacer efectivos los derechos (removiendo las barreras existentes); así como la colaboración y cooperación para hacer frente a los desafíos, lo que conlleva confianza mutua y respeto. Resaltamos la importancia del reconocimiento como un valor central para el desarrollo de una educación más inclusiva, trayendo a colación el pensamiento de Honnet (2010) para asumir que el reconocimiento del otro se ha configurado como una dimensión (y paradigma) central de la justicia social.

Sin duda, todos estos documentos han sido diseñados para abrir procesos de reflexión en los centros comenzando con una autoevaluación o identificación de necesidades a través de técnicas de discusión, visualización de prácticas ideales, cuestionarios y/o rúbricas. A continuación, se pasa a una segunda parte en la que se busca resolver algunos factores susceptibles de mejora. Esto se hace de diferentes formas, a través de casos reales, ejemplos de situaciones, testimonios y evidencias de investigación. A este respecto, destaca que las propuestas de mejora se plantean para la totalidad de estudiantes y no solo para colectivos tradicionalmente vulnerables.

Al igual que en la revisión de Guirao y Arnaiz (2014), casi todas las guías comentadas surgen del ámbito anglosajón, con las diferencias que esto conlleva, lo que indica la necesidad de reforzar esta línea de investigación en nuestro país, confeccionando apoyos prácticos a las comunidades escolares para recorrer juntos el camino inclusivo. Paralelamente, debe incidirse en la necesidad de utilizar y dar a conocer el contenido de este tipo de recursos en contextos de habla hispana, generando espacios de reflexión y debate que contribuyan al avance de este activo pedagógico innegociable.

De la lectura de estos instrumentos se puede concluir que el término inclusión ha superado la condición de acceso a la educación tradicionalmente expuesta en nuestros marcos legales, y busca proporcionar espacios de aprendizaje de calidad y pedagogías que permitan a los estudiantes prosperar, comprender sus realidades y diferencias individuales como una oportunidad para democratizar y enriquecer el aprendizaje. En este sentido, la guía de la UNESCO (2017) invita a ver las diferencias no como problemas a resolver, sino como oportunidades para democratizar y enriquecer el aprendizaje, postura con la que estamos totalmente de acuerdo.

En definitiva, bien podría decirse que un sistema educativo nunca puede ser de calidad si mantiene en su seno mecanismos de naturaleza excluyente. Y dado que, de hecho, los niveles de segregación, marginación y fracaso escolar (tres dimensiones nucleares e interdependientes de la exclusión) siguen siendo altos en los sistemas educativos, no es de extrañar que la meta de una educación inclusiva se haya convertido, internacionalmente también, en la forma de referirse al camino que todos los países deben emprender.

\section{Referencias bibliográficas}

Ainscow, M. (2015). Struggles for equity in education: The selected works of Mel Ainscow. London: Routledge.

(C) Ediciones Universidad de Salamanca / CC BY-NC-ND

Siglo Cero, vol. 50 (3), n. ${ }^{\circ}$ 271, 2019, julio-septiembre, pp. 7-27 
Ainscow, M., Booth, T., Dyson, A., Farrell, P., Frankham, J., Gallannaugh, F., Howes, A. y SMith, R. (2006). Improving Schools, Developing Inclusion. London: Routledge.

Azorín, C. (2016). La respuesta a la diversidad del alumnado en el contexto inglés: un estudio de casos. Enseñanza and Teaching, 34 (2), 77-91.

Azorín, C. (2018a). Percepciones docentes sobre la atención a la diversidad: propuestas desde la práctica para la mejora de la inclusión educativa. ENSAYOS, Revista de la Facultad de Educación de Albacete, 33 (1), 173-188.

Azorín, C. (2018b). The journey towards inclusion: Exploring the response of teachers to the challenge of diversity in schools. Revista Colombiana de Educación, 75, 39-58.

Azorín, C. y Ainscow, M. (2018). Guiding schools on their journey towards inclusion. International Journal of Inclusive Education, 1-19.

Azorín, C., Arnaiz, P. y Maquilón, J. J. (2017). Revisión de instrumentos sobre atención a la diversidad para una educación inclusiva de calidad. Revista Mexicana de Investigación Educativa, 22 (75), 1021-1045.

Barbosa, J. W., Barbosa, J. C. y Rodríguez, M. (2013). Revisión y análisis documental para estado del arte: una propuesta metodológica desde el contexto de la sistematización de experiencias educativas. Investigación Bibliotecológica, 27 (61), 83-105.

Bоотн, T. y Ainscow, M. (2011). Index for Inclusion. Developing learning and participation in schools. Bristol: CSIE.

Booth, T. y Ainscow, M. (2015). Guía para la Educación Inclusiva. Desarrollando el aprendizaje y la participación en los centros escolares. Madrid: Fuhem-OEI.

Booth, T., Simón, C., SAndoval, C., Echeita, G. y Muñoz, Y. (2015). Guía para la Educación Inclusiva. Promoviendo el aprendizaje y la participación en las escuelas: Nueva edición revisada y ampliada. Revista Iberoamericana sobre Calidad, Eficacia y Cambio en Educación, $13(3), 5-19$.

British COUNCIL (2010). Inclusion and diversity in education. Guidelines for inclusion and diversity in schools. Madrid: British Council.

Council of Europe (2015). Tool to Upgrade teacher education practices for inclusive education. Strasbourg: Council of Europe.

CSIE (2015). Equality: Making it happen. A guide for schools to make sure everyone is safe, included and learning. Bristol: Centre for Studies on Inclusive Education.

Department of Children and Youth Affairs (2016). Diversity, equality and inclusion charter and guidelines for early childhood care and education. Dublin: Minister for Children and Youth Affairs.

EcheitA, G. (2006). Educación para la inclusión o educación sin exclusiones. Madrid: Narcea.

EcheitA, G. (2017). Educación inclusiva. Sonrisas y lágrimas. Aula Abierta, 46, 17-24.

EDUCATION SCOTLAND (2015). How good is our school? Livingston: Education Scotland.

Escudero, J. M. (2012). La educación inclusiva, una cuestión de derecho. Educatio Siglo XXI, $30(2), 109-128$

FERNÁNDEZ, F. (2002). El análisis de contenido como ayuda metodológica para la investigación. Revista de Ciencias Sociales, 2 (96), 35-53.

Florian, L. y BEATON, M. (2017). Inclusive pedagogy in action: getting it right for every child. International Journal of Inclusive Education, 1-15.

Göransson, K. y Nilholm, C. (2014). Conceptual diversities and empirical shortcomings - a critical analysis of research on inclusive education. European Journal of Special Needs Education, 29 (3), 265-280.

Guirao, J. M. y Arnaiz, P. (2014). Instrumentos para la autoevaluación y la mejora de la atención a la diversidad en centros educativos. Siglo Cero, 45 (4), 22-47.

(C) Ediciones Universidad de Salamanca / CC BY-NC-ND

Siglo Cero, vol. 50 (3), n. ${ }^{\circ}$ 271, 2019, julio-septiembre, pp. 7-27 
Guirao-Goris, J. A., Olmedo, A. y Ferrer, E. (2008). El artículo de revisión. Revista Iberoamericana de Enfermería Comunitaria, 1, 1-16.

Honneth, A. (2010). Work and recognition: a redefinition. En H. C. Schmidt, A. M. Bush y C. F. ZURN (Eds.), The philosophy of recognition: historical and contemporary perspectives (pp. 223-240). Plymouth: Lexington Books.

IBE y UNESCO (2016). Reaching out all learners. A Resource Pack for Supporting Inclusive Education. Geneva: IBE-UNESCO.

International Baccalaureate Organization (2016). Learning diversity and inclusion in IB programmes. Geneva: International Baccalaureate Organization.

LÓPEZ, F. (2002). El análisis de contenido como método de investigación. XXI, Revista de Educación, 4, 167-179.

López-Vélez, A. L. (2018). La escuela inclusiva: el derecho a la equidad y la excelencia educativa. Euskal Herriko Unibertsitatea: Servicio Editorial de la Universidad del País Vasco.

Loreto, M., López, M. y Assaél, J. (2015). Concepciones docentes para responder a la diversidad: ¿Barreras o recursos para la inclusión educativa? Psicoperspectivas. Individno y Sociedad, 14 (3), 68-79.

Marchesi, A. y Martín, E. (2014). Calidad de la enseñanza en tiempos de crisis. Madrid: Alianza Editorial.

Messiou, K. (2012). Confronting marginalisation in education. A Framework for promoting inclusion. London: Routledge.

Messiou, K. (2017). A Research in the field of inclusive education: Time for a rethink? International Journal of Inclusive Education, 21 (2), 146-159.

Messiou, K. y Ainscow, M. (2015). Responding to learner diversity: student views as a catalyst for powerful teacher development? Teaching and Teacher Education, 51, 246-255.

Miles, S. y Ainscow, M. (2011). Responding to diversity in schools. An inquiry-based approach. London: Routledge.

Muntaner, J. J. (2016). Evaluación y calidad en la educación inclusiva. En I. E. Ramírez (Ed.), Voces de la inclusión. Interpelaciones y críticas a la idea de "inclusión" escolar (pp. 400-430). Buenos Aires: Praxis Editorial.

Murillo, J. y Hernández, R. (2011). Hacia un concepto de justicia social. Revista Iberoamericana sobre Calidad, Eficacia y Cambio en Educación, 9 (4), 8-23.

New Brunkwick Association for Community Living (2011). Creating an inclusive school. indicators of success. A reflection tool for administrators, educators and other school staff. Ottawa: Government of Canada.

New Zealand Ministry of Education (2014). Inclusive practice in secondary schools. Ideas for school leaders. Wellington: New Zealand Ministry of Education.

NguYen, X. T. (2015). The journey to inclusion. Rottherdam: Sense Publishers.

OECD (2018). Preparing our youth for an inclusive and sustainable world. The OECD PISA global competence framework. Paris: OECD.

Peña, T. y Pirela, J. (2007). La complejidad del análisis documental. Información, Cultura y Sociedad, 16, 55-81.

Puig Rovira, J. M., Doménech, I., Gijón, M., Martín, X., Rubio, L. y Trilla, J. (2012). Cultura moral y educación. Barcelona: Graó.

RoJAs, I. R. (2011). Elementos para el diseño de técnicas de investigación: una propuesta de definiciones y procedimientos en la investigación científica. Tiempo de Educar, 12 (24), 227-297.

Simón, C., Echeita, G. y SAndoval, M. (2018). Incorporating students' voices in the "Lesson Study" as a teacher-training and improvement strategy for inclusion. Cultura y Educación, 30 (1), 205-225.

(C) Ediciones Universidad de Salamanca / CC BY-NC-ND

Siglo Cero, vol. 50 (3), n. ${ }^{\circ}$ 271, 2019, julio-septiembre, pp. 7-27 
Simón, C., SANDOVAl, M. y EcheitA, G. (2017). The inclusion journey: Lessons learned from the spanish schools. Copenhagen: European Conference on Educational Research.

SLEE, R. (2013). How do we make inclusive education happen when exclusion is a political predisposition? International Journal of Inclusive Education, 17 (8), 895-907.

The Alberta Teachers' Association (2015). Coaching to support inclusion: a principal's guide. Edmonton: The Alberta Teachers' Associaton.

UNESCO (2015a). Education 2030. Incheon declaration and framework for action. Towards inclusive and equitable quality education and lifelong learning for all. Paris: UNESCO.

UNESCO (2015b). Embracing diversity: Toolkit for creating inclusive, learning-friendly environments. Paris: UNESCO.

UNESCO (2017). Guía para asegurar la inclusión y la equidad en la educación. Paris: UNESCO. 


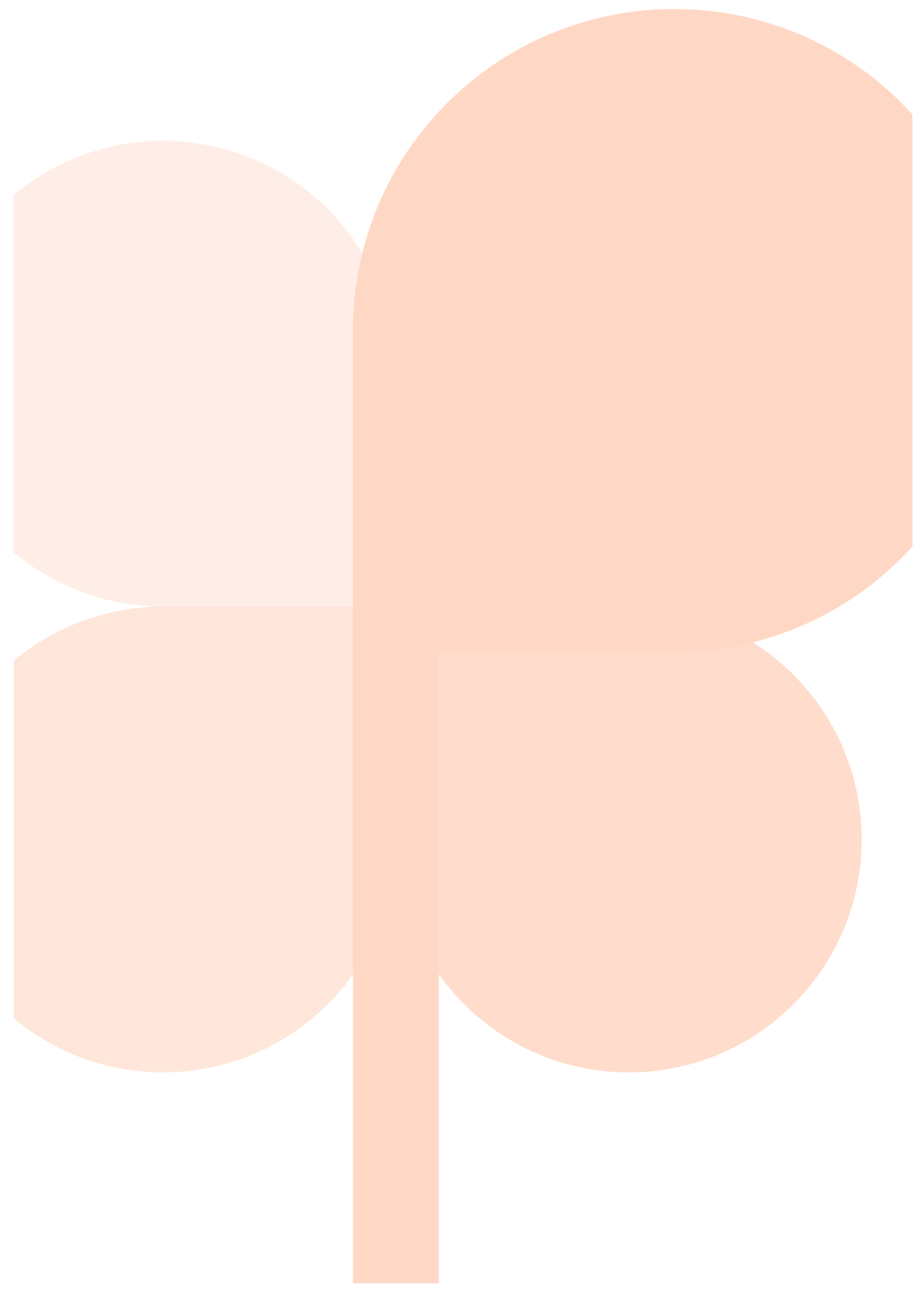

